\title{
O efeito do desoxicolato de sódio no tratamento da gordura localizada: estudo de revisão
}

\author{
The effect of sodium deoxycholate in the treatment of localized \\ fat : review of study
}

\author{
Karen Geremia ${ }^{1}$ \\ Tiago Fontanive ${ }^{2}$ \\ Marcello Mascarenhas ${ }^{3}$
}

\section{RESUMO}

A gordura localizada se caracteriza pela hipertrofia dos adipócitos e está entre as principais queixas na área estética. Nesse sentido, existe um constante desenvolvimento de métodos minimamente invasivos como as infiltrações subcutâneas, que têm intuito de provocar a lipólise das células adiposas. O objetivo do estudo foi realizar uma revisão bibliográfica sobre a ação do desoxicolato de sódio no tratamento da gordura localizada. Para isso, executamos uma revisão bibliográfica que se deu nas bases de indexação PubMed, Scielo, Bireme e LILACS utilizando como descritores:infiltrações subcutâneas, mesoterapia, tratamento minimamente invasivo, gordura localizada e desoxicolato de sódio. Dessa forma foram separados artigos que abordaram o tema em questão, publicados em língua portuguesa, inglesa ou espanholaentre os anos de 2004 a 2015.Identificamoscom base na literatura que o desoxicolato de sódio demonstra uma melhoria em vários perfis de pacientes por destruir os adipócitos e causar uma retração na pele. Foram descritos lipídeos livres, provavelmente originados da lise dos adipócitos, o que não comprometeu a função hepática. Portanto, os estudos pesquisados demonstraram que o desoxicolato de sódio apresenta uma eficácia no tratamento da gordura localizada, porém existe a necessidade de mais estudos referentes a dosagem, além de uma metodologia adequada para a confirmação dos benefícios das infiltrações subcutâneas como ferramenta útil no tratamento da gordura localizada.

\section{PALAVRAS-CHAVE:}

Infiltrações subcutâneas; Mesoterapia; Tratamento minimamente invasivo; Gordura Localizada; Desoxicolato de Sódio.

\footnotetext{
${ }^{1}$ Laboratório de Toxicologia e Mutagênese - Centro Universitário Metodista -IPA. Instituto Cenecista De Ensino Superior De Santo Ângelo - Campanha Nacional de Escolas Da Comunidade - CNEC

${ }^{2}$ Instituto Cenecista De Ensino Superior De Santo Ângelo - Campanha Nacional de Escolas Da Comunidade - CNEC.

${ }^{3}$ Docente do Programa de Pós-Graduação Stricto Sensu em Biociências e Reabilitação.
} 


\section{ABSTRACT}

The localized fat is characterized by hypertrophy of adipocytes and its among the main complaints in the aesthetic area. In this sense, there is a constant development of minimally invasive methods such as subcutaneous infiltrations, which are intended to cause lipolysis of fat cells. The aim of the study was to conduct a literature review on the action of sodium de oxycholate in the treatment of localized fat. Reach this goal, the study performs a bibliographic review, on the indexin gin PubMed ,Scielo, Bireme and LILACS, using as descriptors: subcutaneous infiltration, mesotherapy, minimally invasive treatment, localized fat and sodium de oxycholate. Thus were separate articles that addressed the issue in question, published in Portuguese, English or Spanish between the 2004 to 2015 . We identified based on the literature that sodium deoxycholate d demonstrates an improvement in various profiles of patients to destroy adipocytes and causes a decrease in the skin. Free lipids have been described, probably originated from the lysis of adipocytes, which did not affect liver function.Therefore, studies have surveyed demonstrated efficacy in the treatment of localized fat, but there is a need for of further studies, with a standardized methodology, for confirming the benefits of subcutaneous infiltration as a useful tool in treating localized fat.

\section{KEYWORDS:}

Subcutaneousinfiltration; Mesotherapy. Minimall yinvasiv etreatment. Sodium de oxycholate. 


\section{INTRODUÇÃO}

A expectativa do corpo em relação aos padrões de beleza é o que interliga a maior incidência a tratamentos estéticos, que dispõe de técnicas que auxiliam a enquadrar a população nos padrões impostos pela mídia. Dentre as principais insatisfações físicas do público feminino, a principal é a gordura localizada (ALBUQUERQUE; MACEDO, 2009). Nesse sentido, várias técnicas promissoras vem sendo desenvolvidas, como as infiltrações subcutâneas de substâncias farmacológicas, popularmente conhecida como mesoterapia, que acabam correspondendo as expectativas espera das clinicamente (ALBUQUERQUE; MACEDO, 2009; JUNQUEIRA; CARNEIRO, 2013; POLI NETO; CAPONI, 2007).

As infiltrações subcutâneas consistem na aplicação de injeções de substâncias farmacológicas diluídas e é um método capaz de estimular o tecido que recebe os medicamentos tanto pela ação da punção quanto pela ação dos fármacos (HERREROS; VELHO; MORAES, 2011). Particularmente, as aplicações de desoxicolato de sódio, têm desempenhado um papel lipolítico bem significativo, removendo a gordura localizada indesejada. Esta técnica de mesoterapia com desoxicolato de sódio tem aparecido na literatura médica como responsável pela lipólise química, por ser um detergente iônico, e tem se demonstrado potencial como tratamento minimamente invasivo na redução de gordura localizada(RONTUNDA et al., 2009).

A grande demanda de tratamentos estéticos que visam a redução de medidas, a escassez de informações científicas sobre o tema, os poucos estudos com metodologias mais rigorosas sobre a eficácia e efeitos toxicológicos, fez com que fosse escolhida uma técnica que têm se demonstrado efetiva. Para isso, realizou-se a revisão bibliográfica sobre a influência das infiltrações subcutâneas de desoxicolato de sódio no tratamento da gordura localizada.

\section{PROCEDIMENTOS METODOLóGICOS}

O estudo caracterizou-se como uma revisão bibliográfica, que se deu nas bases de indexação U.S. National Library of Medicine (PubMed), ScientificElectronic Library Online (Scielo) e Biblioteca Virtual em saúde (Bireme), Literatura Latino-Americana e do Caribe em Ciências da Saúde (LILACS) utilizando como descritores: infiltrações subcutâneas; mesoterapia; tratamento minimamente invasivo; gordura localizada; desoxicolato de sódio. Dessa forma foram separados artigos que abordaram o tema em questão, publicados em língua portuguesa, inglesa ou espanholaentre os anos de 2004 a 2015.

\section{RESULTADOS E DISCUSSÃO}

Na década de 60, o Lipostabil@ (Artesan Pharma, Lüchow, Alemanha) desenvolveu uma formulação farmacêutica, para prevenir ou tratar embolia gordurosa, contendo fosfatidilcolina dissolvida em solução com desoxicolato de sódio (KLEIN et al., 2009). No ano de 2001, este medicamento começou a ser usado por via subcutânea, para a dissolução de gorduras (KLEIN et al., 2009; MATARASSO; PFEIFER, 2009; ATIYEH et al., 2008).

A combinação de fosfatidilcolina, um fosfolípido, e seu solvente, o desoxicolato de sódio de sais biliares, têm aparecido na literatura médica como responsáveis pela lipólise química na mesoterapia e tem sido comercializado nos Estados Unidos sob vários nomes. A formulação mais comumente injetada consiste em concentrações variadas de fosfatidil colina (2,5-5,0\%) e desoxicolato de sódio (2,5-4,7\%) (RONTUNDA et al., 2009). Conforme demonstrado, relatado e apontado em estudo, o desoxicolato de sódio, quando administrado isoladamente, por via subcutânea, tem o mesmo efeito que quando associado com fosfatidil colina (Rontundaet al.,2004), de acordo com trabalho posterior, onde foi verificado a lise dos adipócitos e a consequente perda, considerada de leve a moderada, do tecido adiposo (ROTUNDA; KOLODNEY,2006).

Matarasso e Pfeifer (2009) relataram que o desoxicolato de sódio poderia destruir os adipócitos por três formas: como agentes causadores de necrose; por causar mobilização dos ácidos graxos de dentro do adipócito; e, na forma cristalina, causar dano celular pela perfuração das membranas biológicas. Além desses processo, existem três mecanismos de retração da pele que foram observadas: as inflamação difusa intradérmica na pele e angiogênese na região de trata- 
mento, além de ablação focal das gorduras subcutâneas que se estende até a camada basal da derme (DUNCAN et al., 2009). Nestes casos, não houveram relatos de alterações nas enzimas hepáticas ou danos sistêmicos além da região do tratamento imediatamente após a injeção (HEXSEL et al., 2005).

Schuller-Petrovic et al. (2008) monitoraram os níveis plasmáticos de triglicerídeos e os níveis das enzimas hepáticas, in vitro utilizando tecido adiposo e in vivo tanto em ratos, como em humanos, após tratamento com Lipostabil ${ }^{\circ}$. As alterações se deram após 4 semanas, onde a atividade da colinesterase (ChE) aumentou 2,3 vezes, as atividades da alanina aminotransferase (ALT) e aspartate aminotransferase (AST) diminuíram aproximadamente $45 \%$, enquanto que os níveis de triglicerídeos aumentaram quatro vezes e ácidos graxos livres, duas vezes. Concluindo comisso, que os lipídeos livres provavelmente são originados da lise dos adipócitos. Hexsel et al. (2005) e Rotunda et al. (2009) nãocorroboramcomosresultadosobservadospelosautoresSchuller-Petrovic et al. (2008), não observando as alterações hepáticas ou no perfil lipídico. Porém, neste caso, os autores não demonstrarmos parâmetros que foram analisados.

Segundo Rontunda et al. (2009), em contraste com tratamentos cosméticos injetáveis familiares como toxina botulínica e preenchimentos, a mesoterapia utilizando detergentes fisiológicas não é um procedimento "hora do almoço", já que os pacientes devem antecipar reações localizadas e não esperar resultados de lipoaspiração, que produz um episódio de inatividade com um resultado eficaz.

A quantidade administrada e a forma de aplicação da mesoterapia, podemos ocasionar alguns efeitos colaterais (DUNCAN; PALMER, 2008). Rotunda et al. (2009) realizaram tratamentos com desoxicolato injetável em mais de 42 voluntários, e observaram principalmente: edema, eritema, dor, ardor ou sensibilidade no local de aplicação, outros efeitos foram dormência, sensação de aperto, prurido e equimoses. A grande maioria dos efeitos colaterais foram classificados de leves a moderados. Duncan e Chubaty (2006) realizaram estudo com 75 pessoas, e a partir dos efeitos colaterais analisados em parte dos voluntários, concluíram que estes foram transitórios e não afetaram o resultado final, não apresentando qualquer tipo de complicação, perdurando durante vários dias após as injeções e diminuindo gradualmente ao longo de 2 semanas. A redução na concentração de desoxicolato de sódio utilizada neste tipo de tratamento pode minimizar seus efeitos colaterais. Para a manutenção dos efeitos desejados surge a necessidade de associação com outros fármacos, como a luteolina que é um bioflavonóide com ação antiinflamatória, ou até mesmo a utilização de sistemas inteligentes para a liberação controlada do mesmo (DUNCAN; CHUBATY, 2006).

Rotunda et al. (2009) confirmam os dados publicados que o desoxicolato sozinho é tão eficaz na ablação gordura como formulações contendo desoxicolato com fosfatidilcolina, e que ambas as formulações produzidas melhoram a estética da gordura localizada. Ainda não se sabe qual a formulação ideal para sistemas baseados em injeções detergentes para lipólise, mas o desoxicolato demonstra uma meIhoria em vários perfis de pacientes e se sugere que estudos rigorosos das técnicas de dosagem e injeção, bem como seleção adequada do paciente, pode ser necessária para otimizar os resultados. A necessidade de uma compreensão adicional da real utilidade, limitações e associações acabará por determinar o destino de tratamento com detergente injetável para tecido adiposo.

\section{CONCLUSÃO}

Proposto como uma alternativa para esculpir o corpo, reduzir peso e rejuvenescera pele, as infiltrações subcutâneas continuam sendo uma opção crescente no mercado da beleza.Quando se refere a gordura localizada, o desoxicolato de sódio é tóxico para o tecido adiposo, causando a lise dos adipócitos e a remoção permanente do mesmo e traz bons resultados no combate da gordura localizada.

Como um salbiliar, o desoxicolato de sódio é conhecido porte refeitos tóxicos e permutações possíveis de formas físicas e respostas teciduais (mobilização, necrose e efeitos desconhecidos). Existe relatos de alterações relacionadas a ChE, ALT, ASTe triglicerídeos através do monitoramento de níveis plasmáticos, mas esses dados são discutíveis, já que ainda não existem dados clínicos publica dos que incluem 
reagentes padronizados, com dose, injeção, técnica e intervalo de tempo específicos.

Portanto, não existe protocolo de tratamento disponível que permita prever a quantidade de tecido ou gordura que será eliminada comum a solução específica, numa quantidade definida, e injetado a uma profundidade especifica dano tecido subcutâneo. Oskits para infiltrações subcutâneas, incluem vários materiais biológicos, além do desoxicolato de sódio, mas essa ciência pode ser avançada apenas por pesquisas científicas e clínicas. A partir disso, tornar-se-áumaferramentaseguraparaprocedimentoscosméticoscomvalidaçãocientífica.

\section{REFERÊNCIAS}

ALBUQUERQUE, P.J.; MACEDO, C.A. Avaliação do uso da radiofrequência no tratamento da fibrose e gordura localizada no pós-operatório tardio de lipoaspiraçãoabdominal: estudo de caso. 2009, 13 p. Dissertação (Mestrado) - Universidade Tuiuti, Paraná, 2009.

ATIYEH, B.S. et al. Cosmetic mesotherapy: Between scientific evidence, science fiction, and lucrative business. Aesthetic Plastic Surgery, [S.I.], v. 32, n. 6, p. 842-849, 2008.

DUNCAN, D. et al. Refinement of technique in injection lipolysis based on scientific studies and clinical evaluation. Clinics in Plastic Surgery, [S.I.], v. 36, n. 2, p. 195-209, 2009.

DUNCAN, D.I.; CHUBATY, R. Clinical safety data and standards of practice for injection lipolysis: a retrospective study. Aesthetic Surgery Journal, [S.I.], v. 26, n. 5, p. 575$585,2006$.
DUNCAN, D. I.; PALMER, M. Fat reduction using phosphatidylcholine/sodium deoxycholate injections: Standard of practice. Aesthetic Plastic Surgery, v. 32, n. 6, p. 858- 872, 2008.

HERREROS, F.O.C.; VELHO, P.E.N.F.; MORAES, A.M. Mesoterapia: uma revisão bibliográfica. An Bras. Dermatol., [S.I.], v. 86 , n. 1, p. 96-101, 2011.

HEXSEL, D. M. et al. Cosmetic uses of injectable phosphatidylcholine on the face. Otolaryngologic Clinics of North America, v. 38, n. 5, p. 1119-1129, 2005.

JUNQUEIRA, L.C.; CARNEIRO J. Histologia básica. 10. ed. Rio de Janeiro, Guanabara Koogan, 2013.

KLEIN, S.M. et al. In vitro studies investigating the effect of subcutaneous phosphatidylcholine injections in the 3T3-L1 adipocyte model: lipolysis or lipid dissolution? PlasticandReconstructiveSurgery, [S.I.], v. 124, n. 2, p. 419-427, 2009.

MATARASSO, A.; PFEIFER, T.M. Mesotherapy and Injection Lipolysis.Clinics in PlasticSurgery, [S.I.], v. 36, n. 2, p. 181 192, 2009.

POLI NETO, P.; CAPONI, S.N.C. A Medicalização da beleza. Interface - Comunic. Saúde,Educ. Brasil, [S.I.], v. 11, n. 23, p. 569-584, 2007.

ROTUNDA, A.M. et al. Randomized double-blind clinical trial of subcutaneously injected deoxycholate versus a phosphatidylcholine- deoxycholate combination for the reduction of submental fat. Dermatol. Surg., v. 35, n. 1, p. 792803, 2009.

ROTUNDA, A.M.; KOLODNEY, M.S. Mesotherapy and phosphatidylcholine injections: Historical clarification and review. DermatologicSurgery, v. 32, n. 4, p. 465-480, 2006.

SCHULLER-PETROVIC, S. et al. Tissue-toxic effects of phosphatidylcholine/deoxycholate after subcutaneous injection for fat dissolution in rats and a human volunteer. Dermatologic Surgery, v. 34, n. 4, p. 529-542, 2008. 